\title{
Fourth Ventricle Choroid Plexus Xanthogranuloma Causing Hydrocephalus
}

\author{
Craig Ferguson, Simon Walling, Alexander Easton, Jai Jai Shiva Shankar
}

Keywords: MRI, Neuropathology, Neuroradiology, Neurosurgery, Tumors

doi:10.1017/cjn.2015.289

Can J Neurol Sci. 2015; 42: 454-456

Xanthogranulomas of the choroid plexus are discovered incidentally on autopsy in 1.6 to $7 \%$ of cases, and are typically found in the lateral ventricles. Very rarely, however, they can become symptomatic because of obstruction of the ventricular system, and these tend to be found in the third ventricle. ${ }^{1}$ There has only been one published case of fourth ventricle choroid plexus xanthogranuloma. ${ }^{2}$ We present a second case to add to the sparse literature of a symptomatic choroid plexus xanthogranuloma of the fourth ventricle causing hydrocephalus. This case demonstrates the difficulty of differentiating these lesions from other intracranial masses including aneurysms.

\section{Case Report}

A middle-aged patient presented with a 6-month history of deterioration in right visual field and a pressure feeling in the center of her forehead. An unenhanced computed tomography (CT) scan of the head (Figure 1A) demonstrated a $9 \times 6 \mathrm{~mm}$ focus of high density in the fourth ventricle. It was thought to be a fourth ventricle calcified meningioma, unrelated to the patient's symptoms. Four months later, magnetic resonance imaging (MRI) (Figure 1B, C) and magnetic resonance angiogram of the head (Figure 1D) performed because of persistent visual symptoms demonstrated the lesion to be T1- and T2-hypointense and nonenhancing in the inferior fourth ventricle. There was no restriction seen on diffusion-weighted imaging. The proximity of this lesion to the distal loop of the left posterior inferior cerebellar artery (PICA) raised the suspicion of an aneurysm. No aneurysm could be seen on the cerebral angiogram performed, leading to suspicion of a thrombosed PICA aneurysm. The lesion continued to be stable on multiple follow-up imaging. Other differential diagnoses considered at this stage were an unusual cystic or other extracranial mass with paramagnetic properties, a desiccated calcified epidermoid cyst, and a calcified subependymoma.

Two years after the initial presentation, this patient presented to the emergency department with nausea and vomiting for 9 days, a slight headache, but no neck stiffness, fever, or photophobia. Her physical examination was unremarkable. An unenhanced CT of the head (Figure 1E, F) demonstrated enlargement of the third and lateral ventricles with no significant change in this lesion.

A preoperative MRI (Figure 1G) demonstrated that the lesion contained a new cystic component, likely representing a subacute hemorrhage. In addition, the nodular component had increased in size, but continued to be hypointense on T2/fluid-attenuated inversion recovery. The patient underwent surgery with near-complete resection of the lesion with relief in her symptoms.
On histopathological examination, there were numerous cholesterol clefts surrounded by inflammatory cells, including mononuclear cells and foamy and hemosiderin laden macrophages, embedded in fibrous tissue as well as scattered multinucleated giant cells (Figure 2). The mass is closely associated with normal choroid plexus epithelium (Figure 2, inset). The features are those of a xanthogranuloma arising from the choroid plexus of the fourth ventricle with no neoplastic elements (Figure 2).

The patient did not have any recurrence of this lesion at 2-year follow-up at the time of this report (Figure 1H).

\section{DISCUSSION}

The first case of a choroid plexus xanthogranuloma was reported in 1900, by Blunner as "cholesteatomatous endothelioma." Since then, choroid plexus xanthogranulomas have been reported incidentally in up to $7 \%$ of autopsy studies, found mostly in the lateral ventricles. ${ }^{1}$ They are more likely to become symptomatic when present in the third ventricle from obstruction of the foramen of Monro or aseptic meningitis from xanthomatous debris spilling into cerebrospinal fluid, causing hydrocephalus. ${ }^{3}$ The fourth ventricle lesion may become symptomatic through similar mechanisms, although little information exists given how exceedingly rare they are in this location.

Xanthogranulomas are benign tumors composed of cholesterol clefts, macrophages, hemosiderin deposits, and multinucleated giant cells in fibrous tissue. ${ }^{4}$ They are thought to arise when desquamative epithelium of choroid plexus enters into the stroma at the site of basal lamina and tubular wall disruption where the lipid content of epithelial cells accumulates as they degenerate and become foamy. Disintegration of the lipid-laden cells releases additional lipid, provoking a macrophage response. Some xanthogranulomas are associated with degenerative changes, including demyelination of neuronal tissues, whereas others form masses despite their nonneoplastic character. This, however, is still a matter of controversy ${ }^{3}$ and the pathogenesis of lateral ventricular xanthogranulomas may be different. ${ }^{4}$

From the Department of Radiology, Division of Neuroradiology, Halifax Infirmary Hospital, Nova Scotia, Canada (JJSS); Faculty of Medicine and Dentistry, University of Alberta, Edmonton, Alberta, Canada (CF); Division of Neurosurgery, Halifax Infirmary Hospital, Halifax, Nova Scotia, Canada (SW); Department of Pathology, Halifax Infirmary Hospital, Halifax, Nova Scotia, Canada (AE).

Received December 15, 2014. Final Revisions Submitted May 11, 2015. Correspondence to: Jai Shankar, QE II Hospital, Radiology, Division of Neuroradiology, 1796 Summer Street, Halifax, Nova Scotia, Canada B3H 3A6. Email: shivajai1 @ gmail.com 


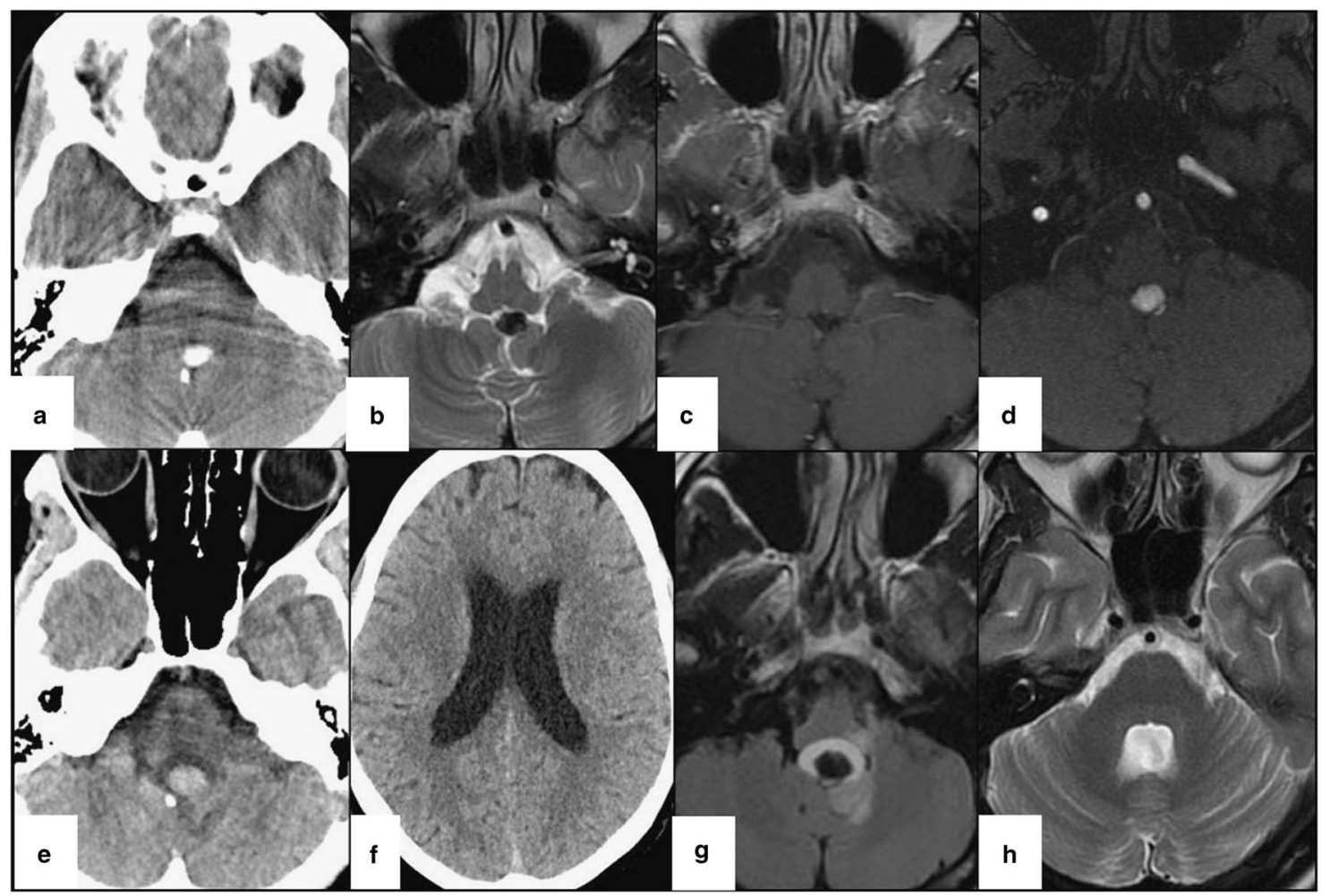

Figure 1: (A) CT of the head (axial $5 \mathrm{~mm}$ ). A $9 \times 6 \mathrm{~mm}$ focus of high density in the fourth ventricle. (B) MRI (1.5 Tesla), axial T2. There is a T2 hypointense and nonenhancing lesion in the inferior fourth ventricle. (C) MRI (1.5 Tesla; axial). TI with gadolinium showing the nonenhancing lesion. (D) The proximity of this lesion to the distal loop of left PICA raised the suspicion of an aneurysm. (E) CT of the head. No change in the posterior fossa lesion. $(F)$ The presence of lateral and third and fourth ventricle hydrocephalus likely resulting from a hemorrhage causing obstructive hydrocephalus. (G) MRI (1.5 Tesla). Axial fluid-attenuated inversion recovery. The lesion contains a new cystic component, likely representing a subacute hemorrhage. The nodular component has increased in size, but continues to be hypointense on T2/fluid-attenuated inversion recovery. (H) MRI (1.5 Tesla), axial T2 propeller. This MRI performed 2 years after surgery demonstrates no tumor recurrence.

CT findings of choroid plexus xanthogranulomas in the literature are variable from hypo-, iso-, and hyperdense, representing the heterogeneity in the content of these lesions. They are round and smooth-walled. Some show contrast enhancement and some have a hypodense central area. MRI of third ventricle xanthogranulomas demonstrate $\mathrm{T} 1$ iso-hyperintensity and $\mathrm{T} 2$ hyperintensity and with some cases demonstrating enhancement following gadolinium. Some cases demonstrate brain edema relating to the xanthogranuloma. ${ }^{4}$ In addition, T2 hypointensity has been demonstrated in symptomatic lateral ventricle xanthogranulomas. ${ }^{5}$

Our case is only the second case reported, and the first since 1985 , of a symptomatic fourth ventricle choroid plexus xanthogranuloma. This resembled an aneurysm, leading physicians down the wrong pathway. The diagnosis is incredibly difficult given the heterogeneity in their appearance and so they can resemble a calcified meningioma, a choroid plexus papilloma, a calcified epidermoid cyst, calcified subependymoma, cavernous malformation, or other cystic lesions such as a colloid cyst. This case adds yet another piece of information to the diverse MRI findings of xanthogranulomas and the diagnostic dilemma that results from it.

Our patient likely developed nausea, vomiting, and headache from a mild hemorrhage from this lesion that caused an obstructive hydrocephalus.

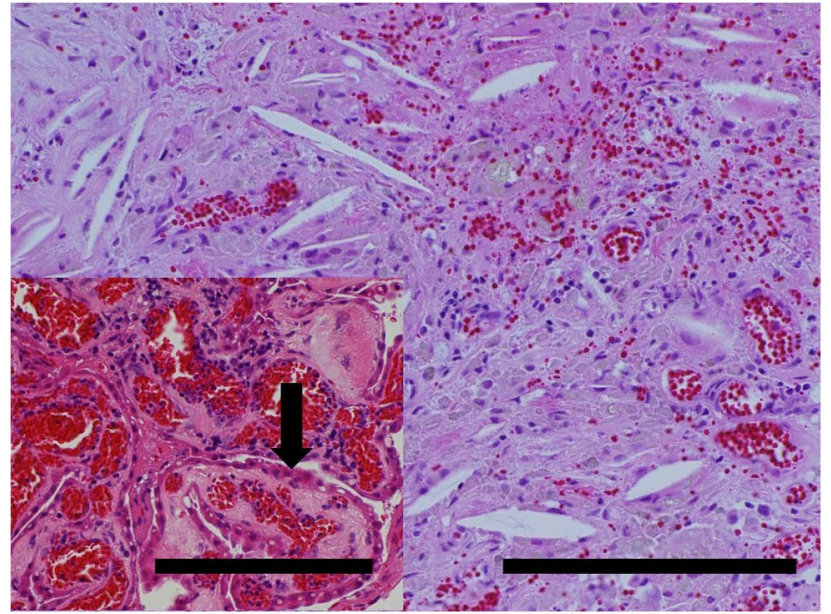

Figure 2: Histopathology of the lesion. The main body of the figure showscholesterol clefts (empty cleft-like spaces; the cholesterol removed during processing), embedded in collagen containing macrophages (some of which contain brown hemosiderin), multinucleated giant cells, and mononuclear inflammatory cells. Inset (lower left) shows fragments of normal choroid plexus epithelium adjacent to the mass (arrow points to typical cobblestoned epithelial lining). Both scale bars equate to 100 microns. 


\section{ACKNOWLEDGMENTS AND Funding}

No funding was received for this project and there are no competing interests.

\section{Disclosures}

None of the authors has anything to disclose.

\section{REFERENCES}

1. Pear BL. Xanthogranuloma of the choroid plexus. AJR Am J Roentgenol. 1984;143:401-2.
2. Wang PY, Hung TP, Huang KM, et al. Xanthogranuloma of the fourth ventricle - a case report. Taiwan Yi Xue Hui Za Zhi. 1985; 84:1386-90.

3. Shetty J, Devadiga KV. Chandrika, et al. Unusual presentation of xanthogranuloma of the choroid plexus. J Neurosci Rural Pract. 2010;1:97-8.

4. Miranda P, Lobato RD, Ricoy JR, et al. Xanthogranuloma of the choroid plexus of the third ventricle: case report and literature review. Neurocirugia (Astur). 2005;16:518-22.

5. Kadota T, Mihara N, Tsuji N, et al. MR of xanthogranuloma of the choroid plexus. AJNR Am J Neuroradiol. 1996;17: 1595-7. 